\title{
5G Promotes the Intelligent Development of the Logistics Industry
}

\author{
Chao Duan \\ Guangzhou College of Technology and Business, Guangzhou, China
}

Keywords: 5G, Communication technology, Logistics, Smart logistics

\begin{abstract}
China's 5G vendors used to announce the official launch at the China International Communications Expo 2019. This initiative, coupled with the continuous coverage plan of 5G base stations in major cities, marks China's beginning to enter the era of $5 \mathrm{G}$ communication services for all commercial people. As a new generation of information and communication technology, 5G's commercialization supports, promotes, and promotes smart logistics that realizes digitization, real-time, automation, visualization, and intelligent tracking and control, empowering the development of smart logistics. This paper explores the development of logistics intelligence by analyzing the impact of 5G technology on application scenarios of digital logistics and intelligent warehousing, automated transportation and logistics tracking, and the realization of smart logistics supply chain based on $5 \mathrm{G}$ technology.
\end{abstract}

\section{Introduction}

"Smart Logistics", the Intelligent Logistics System, was first proposed by IBM. With the continuous development of information technology and the continuous improvement of logistics management level, the China Logistics Technology Association Information Center and other organizations emphasized the concept jointly proposed by "Smart Logistics" Use intelligent technology and means to improve the level of intelligence and automation of the logistics system [1]. The key to the development of smart logistics lies in the applications of big data, artificial intelligence, autonomous driving, and the Internet of Things, and the characteristics of $5 \mathrm{G}$ communication technology with ultra-low latency, high-speed broadband, and massive access can promote big data, artificial intelligence, autonomous driving, and physical The technological progress of networking has further empowered the development of intelligent logistics. Intelligent construction is essential for the development of the logistics industry, which can enable modern logistics to operate more efficiently, save labour costs, and respond quickly to meet customer needs. Therefore, seize the opportunity of $5 \mathrm{G}$ commercialization and use the new generation of $5 \mathrm{G}$ information technology to become a development the key to smart logistics. Based on this, this article will analyse the impact of $5 G$ technology on the application scenarios of digital logistics, intelligent warehousing, automated transportation and logistics tracking and the realization of supply chain smart logistics [1].

\section{5G Empowers Digital Logistics}

$5 \mathrm{G}$ is the fifth-generation communication technology. As shown in Figure 1, it has three characteristics of ultra-low latency, high-speed broadband and massive access. Its theoretical speed is $10 \mathrm{~GB} / \mathrm{s}$, which can reach 100 times the speed of 4G, and its stability can reach $99.999 \%$. And the delay is less than $10 \mathrm{~ms}$. These characteristics mean that the application of $5 \mathrm{G}$ makes data acquisition and transmission more massive, fast and accurate than 4G communication technology. It also means that $5 \mathrm{G}$ can help the logistics industry to use historical logistics data for analysis and improvement to achieve real-time data scheduling, and then realize data collaboration in all aspects of logistics [2]. 


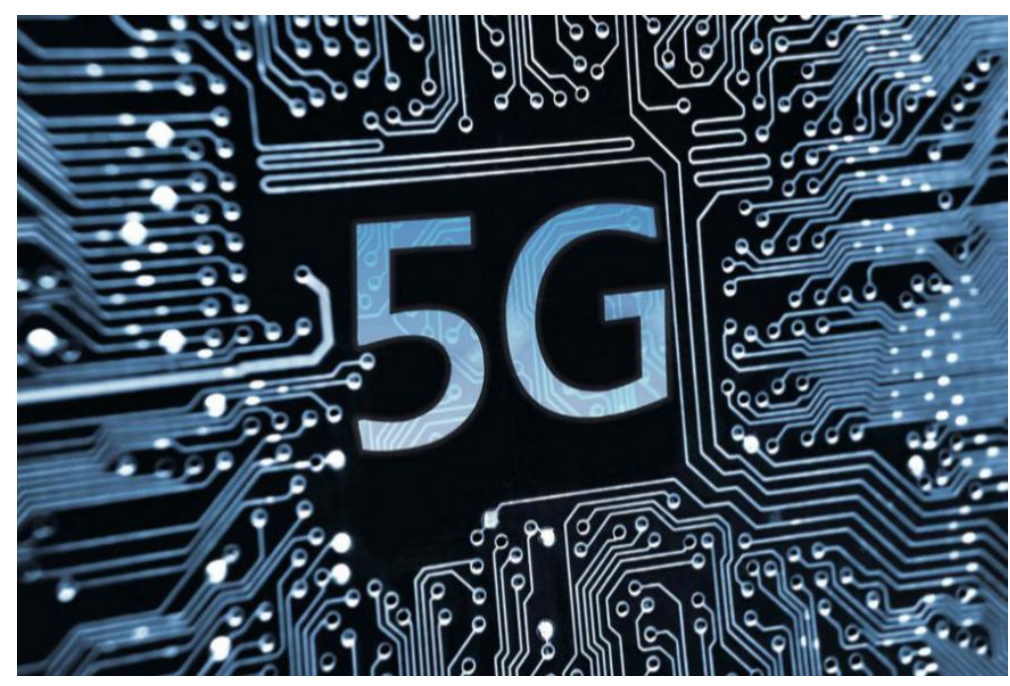

Fig.15G Communication Technology

Digitalization is an opportunity for the development of the logistics industry and the centre of the development strategy of the logistics industry. Digital logistics is not only reflected in the digitization of logistics product information, but also the digitization of all elements of the logistics system, including the digitization of warehouse equipment and operations, the digitization of transportation vehicles and scheduling, and packaging And information processing digitization. The digitization of logistics can significantly enhance the competitiveness of enterprises in the market, and the flow of logistics information through various links can be optimized. At the same time, the operational efficiency will be higher and more transparent [3]. Digital logistics will speed up the processing of orders, realize the automation of order processing and delivery, and dynamically optimize transportation routes.

Digital logistics can be called "cloud logistics". The massive information storage and efficient information analysis capabilities of modern "cloud logistics" mainly depend on the application of big data and cloud computing. The characteristics of 5G high-speed broadband can not only further improve "cloud logistics" the practicality of the architecture can also provide high-speed communication for edge computing, thereby further optimizing a new generation of logistics computing solutions, and its massive access feature can also enable centralized computing and edge computing to seamlessly integrate. In the application scenario of modern logistics, most of the nodes are edge communication nodes [3]. 5G access to the new generation of logistics will realize that all mobile nodes of logistics can put data calculation, storage, cache, etc. at the edge of the terminal network, and then the edge server is responsible Synchronize data with remote data cloud computing communications, and at the same time reduce resource consumption caused by redundant computing and data transmission.

\section{5G Promotes Comprehensive and Intelligent Logistics Environment}

Warehousing management is an important link in logistics. In the traditional logistics warehousing management, the operations of storage, storage, loading and unloading, and delivery of goods are mainly manpower-based. It is difficult to achieve the efficiency of the logistics warehousing process, and face the increasing labour force. Costs and safety issues in the handling of large items, logistics companies have continuously increased the use of logistics equipment, and gradually improved the degree of automation in the warehousing process. Automation and intelligent warehousing have emerged. Modern logistics mainly relies on the intelligent warehouse management system to realize the intelligent warehouse [4]. The design of the intelligent warehouse management system is mainly supported by RFID technology, which is endowed with barcodes and encodes the inventory, shelves and pallets in the warehouse, so as to realize intelligent warehouse management. In the new generation of smart logistics, fully automated logistics and warehousing links rely on the application of artificial intelligence technology to achieve intelligent storage operations through artificial 
intelligence technology, such as the use of intelligent robots in the storage carrier execution layer to help achieve item storage, item transmission and item [4].

5G communication technology makes the operation of the intelligent logistics warehousing information system more efficient and stable, making the use of drones, robots, unmanned shuttles, AR glasses, and automatic sorting equipment in the carrier execution level more reliable, and at the same time can realize the execution level the decentralization of data transmission, when the system is running, the data source at the end of the execution layer is directly uploaded to the data cloud for processing, and then the system instructions and operation instructions are issued downwards to promote the logistics warehousing process from the arrival, inspection, picking, inventory, and packaging of goods And delivery and other operations to achieve a comprehensive data warehousing environment, visualization and automation, as shown in Figure 2. The use of robots, unmanned shuttles, and automatic sorting equipment has streamlined manual operations, greatly reduced labour costs, and to a certain extent, ensured the safety of warehouse operations, optimized item inquiries, and improved outbound and inbound storage efficiency, easy for systematic management of warehouse [5].

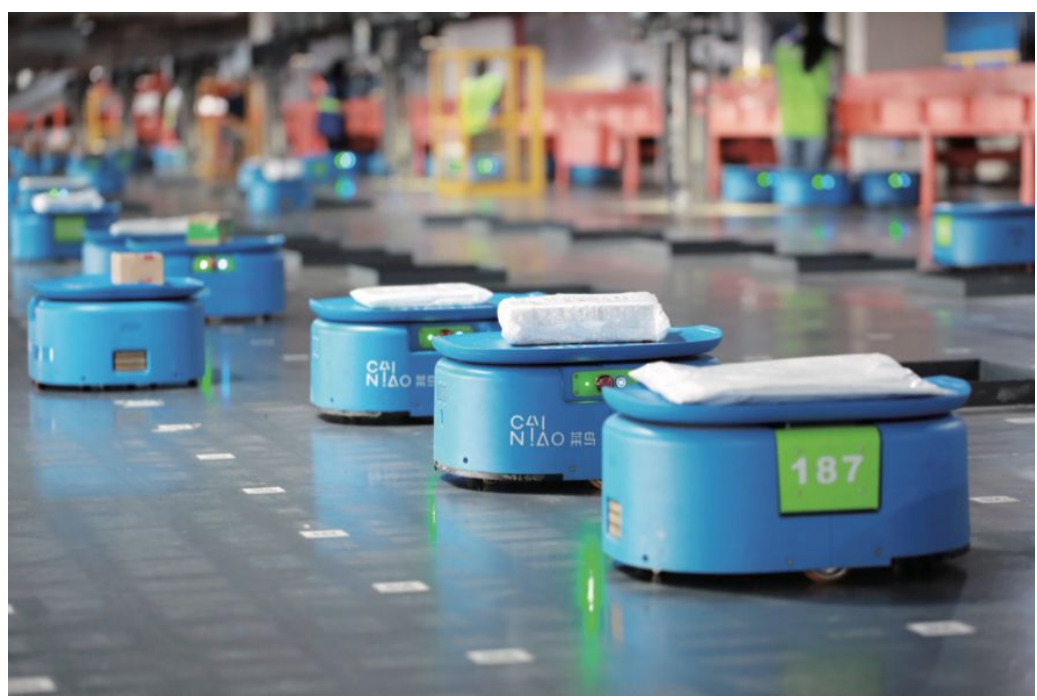

Fig.2 Application of 5G in Logistics

\section{5G Promotes Fully Automatic Logistics Transportation and Distribution}

Modern transportation methods are mainly railway transportation, road transportation, water transportation, air transportation and pipeline transportation. The transportation and distribution process faces challenges such as labour cost, driving safety and dispatch accuracy. In order to improve the efficiency of logistics transportation and distribution, ensure the safety of transportation and distribution and reduce the human cost of transportation and distribution, the modern logistics industry is constantly exploring the full automation of logistics and transportation [5].

The fully automatic realization of the logistics transportation and distribution link needs to be combined with the use of the Internet of Vehicles system. This system has a real-time live view function. By installing on-board terminal equipment on the vehicle dashboard, it can collect, store and send all the working conditions and static and dynamic information of the vehicle. , Using the mobile network to achieve human-vehicle interaction. 5G can meet the needs of the Internet of Vehicles environment, such as self-organizing network construction, real-time data sharing, massive transmission, low latency and high reliability, and other high-quality performance, which can promote automated transport and distribution and unmanned driving scenarios supported by Internet of Vehicles technology in smart logistics. 5G is mainly used for terminal communication in logistics transportation and distribution. Its low-latency feature allows the vehicle status and road conditions of transportation vehicles at the logistics end of the logistics transportation and distribution link to interact with the remotely controlled cloud data and communicate more quickly. To achieve end-to-end seamless connection, thereby improving the efficiency of the remote control centre for scheduling planning [6]. 


\section{5G Guarantee Logistics Intelligent Tracking}

Logistics tracking refers to the use of certain information technology to realize the full tracking and timely monitoring of the location, quantity, quality, vehicle configuration and other information of the logistics products during the storage and transportation of logistics products in all aspects of logistics. Existing logistics monitoring system designs are mostly based on wireless radio frequency technology and GPRS technology to design the labels of logistics items to be monitored, and then use GPRS technology to identify the labels, and collect, store and transmit the label information to achieve logistics Better monitoring function. The new generation of logistics tracking combines cutting-edge technologies such as big data, cloud computing and artificial intelligence, and uses 5G, a stable, ultra-low latency, high-speed broadband and massive access communication technology, to further realize the temperature and humidity environment of logistics products [6].

The main functional modules of the logistics monitoring system based on 5G are composed of information acquisition module, database management module and GIS information platform. Among them, the information collection module mainly collects product information, environment awareness information and GPS position information, the database management module mainly manages basic geographic location information and product logistics information, and the GIS information platform is used to realize statistical analysis, information query, display control and real-time monitoring [7]. Facing the abnormal situation of the logistics monitoring system, 5G can help the abnormal problems to upload the data such as pictures and videos to the data processing centre of the system for efficient processing, and make intelligent decisions and remote intelligent guidance based on the processing results, not only to maintain the safety of logistics transportation , Can also reduce the loss of items due to changes in temperature and humidity during the cold chain transportation, so compared with traditional communication technology, 5G can ensure that the new generation of logistics monitoring system is more efficient and intelligent, to ensure the safety of items and reduce costs, so as to truly achieve instant track [7].

\section{5G Escort Smart Logistics Supply Chain}

The innovative smart logistics business model is more digital, automated, and intelligent than the traditional logistics business model, enabling the coordination of all logistics operations. The development of smart logistics should not only focus on the enterprise itself, but also on the coordination with the cooperative enterprise, and use the supply chain. Management concepts and models to achieve more systematic and integrated management to solve the problem of high logistics operation costs in all aspects of logistics, and through the information flow, capital flow and business flow between various upstream and downstream enterprise nodes and consumers of logistics enterprises Information communication to further improve the efficiency of logistics operations, grasp dynamic market changes and quickly respond to customer needs, and enhance the core competitiveness and efficiency of enterprises [8].

The key to realizing the development of intelligent logistics supply chain lies in the integration and collaborative management between logistics enterprises and upstream and downstream [8]. The problem of how to store data effectively, analyse it accurately, and transmit it efficiently can only help companies make effective decisions and achieve collaborative management throughout the supply chain. The smart logistics supply chain business model can rely on a smart logistics integrated service platform. The application layer includes a warehousing management system, a procurement management system, a partner management system, an internal OA, financial and human resources system. The underlying technology is still inseparable from big data, Cloud computing, Internet of Things and artificial intelligence [9]. Based on the three characteristics of ultra-low latency, high-speed broadband and massive access based on 5G communication technology, it can escort the stable and efficient operation of the intelligent logistics integrated service platform, help solve the data problems facing the intelligent logistics supply chain, and build a more robust and efficient operation And a smart logistics business model with collaborative processing capabilities. 5G enables highly efficient sharing of logistics supply chain data, turning all node enterprises into a whole, realizing strategic integrated management of the whole process, mastering dynamic market 
information, realizing all aspects of the supply chain to respond to customer needs in an instant, collaboratively meet customer needs, purchase, Warehousing, transportation, inventory, after-sales profit maximization and cost minimization [9].

\section{Summary}

In summary, the commercialization of $5 \mathrm{G}$ communication technology will have a significant impact on the development of the logistics industry and will further accelerate the process of intelligent development of logistics. China's logistics industry is developing well, and technologies such as big data, cloud computing, the Internet of Things, and artificial intelligence are maturing. The government is promoting the development of enterprise supply chains and attaching importance to the continuous reform and upgrade of the logistics industry. Facing this historical opportunity, we use $5 \mathrm{G}$ communications the three major characteristics of technology combined with the concept of supply chain management, let the new generation of logistics develop in the direction of digital, automated, intelligent and supply chain smart logistics, provide consumers with higher quality logistics services, and smart logistics will also Lead and popularize 5G applications.

\section{Acknowledgements}

This paper is supported by Guangdong Youth Fund Project, No. 2019KQNCX232.

\section{References}

[1] Chen, Y.J. Analysis of the Application in the 5G era. The World Of Electronic Products, no.11, pp.15-17,2019.

[2] Liang, R.W. Research on Logistics Warehouse Management Based on Internet of Things. Modern Marketing, no.12, pp.121-123,2019.

[3] Liu, N.T. Dou, Z.W. On the Information Development of Intelligent Logistics Warehousing in the 5G Era. Logistics Engineering and Management, no. 6, pp. 3-7, 2019.

[4] Li, J.T. Design Real-Time Monitoring System of Cold Chain Logistics Based on Radio Frequency Identification Technology and GPRS Technology. Automation and Instrumentation, no.7, pp.88-90, 2019.

[5] Yang, X.J. Design and Implementation of the Intelligent Logistics Transportation Management Service System. Digital Technology and Application, no.3, pp.188-189, 2019.

[6] Luo, Y.H., Lin, N. Research on the Development of Smart Logistics Business Model Based on Supply Chain Perspective. Business Economic Research, no.21, pp.82-85, 2019.

[7] Zeng, W.L., Wu, M.S. Sun, W.J. A Review of Research on Autonomous Taxi Dispatching Systems. Computer Science, no.1, pp.15-17, 2020.

[8] Zhou, Z.J. Innovative Analysis of Supply Chain Logistics Enterprise Management Model. Modern Marketing, no.1, pp.119-122, 2020.

[9] Wan, L.T. The Next 3 to 5 Years will be a Critical Period for the Intelligentization of Logistics in China. Entrepreneur Forum, no.12, pp.58-61, 2017. 